\title{
Proof of a conjecture of Selmer
}

\author{
by \\ JukKa PIHKo (Helsinki)
}

1. Introduction. The following definitions, results, and conjecture are taken from [7], to which the reader is referred for background.

Let $k \geq 1$ be an integer. Consider a basis $A_{k}=\left\{a_{1}, \ldots, a_{k}\right\}$, where $1=a_{1}<\ldots<a_{k}$ are integers ("stamp denominations"). Let $h \geq 1$ be an integer ("size of the envelope"). Consider all combinations

$$
x_{1} a_{1}+\ldots+x_{k} a_{k}, \quad \text { where } x_{i} \geq 0 \text { are integers and } \sum_{i=1}^{k} x_{i} \leq h .
$$

Let $N_{h}\left(A_{k}\right)=$ smallest positive integer not represented by (1.1) and let $n_{h}\left(A_{k}\right)=N_{h}\left(A_{k}\right)-1=$ the $h$-range of $A_{k}$. To emphasize the role of $h$ in these considerations, we will call $A_{k}$ an $h$-basis in this paper. Let $h_{0}=$ $h_{0}\left(A_{k}\right)=\min \left\{h \mid n_{h}\left(A_{k}\right) \geq a_{k}\right\}$.

Remark 1.1. The importance of $h_{0}$ stems from the fact that we are (basically) interested only in the case $h \geq h_{0}$. For if $h<h_{0}$, then in the representations (1.1) of the integers $n, 1 \leq n \leq n_{h}\left(A_{k}\right)$, we cannot use $a_{k}$ at all.

The "local" problem: given $h$ and $A_{k}$, determine $n_{h}\left(A_{k}\right)$. The "global" problem: given $h$ and $k$, find the extremal $h$-range $n_{h}(k)=\max \left\{n_{h}\left(A_{k}\right)\right\}$ and extremal $h$-bases $A_{k}^{*}$ such that $n_{h}\left(A_{k}^{*}\right)=n_{h}(k)$.

If, in addition to (1.1), we have

$$
x_{1} a_{1}+\ldots+x_{j} a_{j}<a_{j+1} \quad \text { for } j=1, \ldots, k-1,
$$

then we have a regular representation, introduced by Hofmeister [1]. Thinking of stamps, (1.2) means that we use first the largest stamp $a_{k}$ as often as possible, then the next stamp $a_{k-1}$ as often as possible, etc.

We can then define analogously the regular h-range $g_{h}\left(A_{k}\right), \widetilde{h}_{0}=\widetilde{h}_{0}\left(A_{k}\right)$ $=\min \left\{h \mid g_{h}\left(A_{k}\right) \geq a_{k}\right\}$, extremal regular $h$-range $g_{h}(k)$ and extremal regular $h$-bases $\widetilde{A}_{k}^{*}: g_{h}\left(\widetilde{A}_{k}^{*}\right)=g_{h}(k)$. In this paper, only regular representations will be considered. 
Since the case $k=1$ is trivial, and also the case $k=2$ is under control (see [7, p. 9.1]), we suppose, in this section, that $k \geq 3$. We denote by $\lfloor x\rfloor$ the greatest integer $\leq x$ and by $\lceil x\rceil$ the least integer $\geq x$ (so that $\lceil x\rceil=-\lfloor-x\rfloor$ for all $x \in \mathbb{R}$ ).

The following result of Hofmeister [1] (see [7, p. 9.1]) solves the local problem in the regular case.

THEOREM 1.2 (Hofmeister). If $h \geq \widetilde{h}_{0}$, then

$$
g_{h}\left(A_{k}\right)=\mu_{1} a_{1}+\ldots+\mu_{k} a_{k}
$$

where the coefficients $\mu_{j}$ are given recursively by

$$
\begin{gathered}
\mu_{j}=\left\lfloor\frac{a_{j+1}-2-\sum_{i=1}^{j-1} \mu_{i} a_{i}}{a_{j}}\right\rfloor \text { for } j=1, \ldots, k-1, \\
\mu_{k}=h-\sum_{i=1}^{k-1} \mu_{i} .
\end{gathered}
$$

The representation (1.3) is regular. We also have

$$
\widetilde{h}_{0}=\sum_{i=1}^{k-1} \mu_{i}+1 .
$$

It follows immediately from (1.5) and (1.6) that

$$
h=\widetilde{h}_{0} \quad \text { if and only if } \quad \mu_{k}=1 .
$$

Suppose now that we are given a sequence $\left(\nu_{1}, \ldots, \nu_{k}\right)$ of nonnegative integers with $\nu_{k} \geq 1$ and

$$
\nu_{1}+\ldots+\nu_{k}=h
$$

We can define a basis $A_{k}=\left\{a_{1}, \ldots, a_{k}\right\}$ by

$$
a_{i+1}=\left(\nu_{i}+2\right) a_{i}-a_{i-1} \quad \text { for } i=1, \ldots, k-1 \quad\left(a_{0}=0\right) .
$$

It is then easy to prove by induction that

$$
\mu_{i}=\nu_{i} \quad \text { for } i=1, \ldots, k-1 .
$$

In fact, if we suppose that $\mu_{i}=\nu_{i}$ for $i=1, \ldots, j-1$, then we have, by (1.9) and (1.4),

$$
\begin{aligned}
\mu_{j} & =\left\lfloor\frac{\left(\nu_{j}+2\right) a_{j}-a_{j-1}-2-\sum_{i=1}^{j-1} \nu_{i} a_{i}}{a_{j}}\right\rfloor=\left\lfloor\frac{\left(\nu_{j}+1\right) a_{j}-a_{1}}{a_{j}}\right\rfloor \\
& =\left(\nu_{j}+1\right)-\left\lceil\frac{a_{1}}{a_{j}}\right\rceil=\nu_{j} .
\end{aligned}
$$

By (1.6) and (1.8) with $\nu_{k} \geq 1$, we have $h \geq \widetilde{h}_{0}$, and using Theorem 1.2 we get $\mu_{k}=\nu_{k}$ and (1.3). 
The following result (see [7, p. 9.5]), conjectured by Hofmeister [1] and proved by Mrose [4, p. 68], solves the global problem completely in the regular case.

Theorem 1.3 (Mrose). The two sets of coefficients

$$
\begin{aligned}
& \mu_{i}^{(1)}= \begin{cases}\left\lfloor\frac{i h}{k}\right\rfloor-\left\lfloor\frac{(i-1) h}{k}\right\rfloor & \text { for } i=1, \ldots, k-1, \\
\left\lceil\frac{h}{k}\right\rceil & \text { for } i=k,\end{cases} \\
& \mu_{i}^{(2)}= \begin{cases}\left\lceil\frac{h}{k}\right\rceil-1 & \text { for } i=1, \\
\left\lceil\frac{i h}{k}\right\rceil-\left\lceil\frac{(i-1) h}{k}\right\rceil & \text { for } i=2, \ldots, k-1, \\
\left\lfloor\frac{h}{k}\right\rfloor+1 & \text { for } i=k,\end{cases}
\end{aligned}
$$

give the extremal regular $h$-bases $\widetilde{A}_{k}^{*}$ through

$$
a_{i+1}=\left(\mu_{i}+2\right) a_{i}-a_{i-1} \quad \text { for } i=1, \ldots, k-1 \quad\left(a_{0}=0\right),
$$

and the extremal regular h-range through (1.3). The two bases are equal if and only if $\operatorname{gcd}(h, k)=1$.

For ordinary $h$-ranges $n_{h}\left(A_{k}\right)$, the ("classical") extremal problem is much more difficult. It is simple only for $k=2$, but very complicated already for $k=3$, a case which was settled by Hofmeister [3]. He also solved the analogous problem, already suggested by Salié [6], of determining the bases $A_{3}$ for which $n_{h_{0}}\left(A_{3}\right)$ is extremal for given $h_{0}$.

Inspired by this, Selmer [7, p. 9.7] raised the analogous problem for regular $h$-ranges. That is, given $h$ and $k$, we define

$$
\mathcal{A}=\left\{A_{k} \mid \widetilde{h}_{0}\left(A_{k}\right)=h\right\} \quad \text { and } \quad g_{\tilde{h}_{0}=h}(k)=\max \left\{g_{h}\left(A_{k}\right) \mid A_{k} \in \mathcal{A}\right\} .
$$

Supported by extensive computer calculations by Svein Mossige, Selmer formulated the following

Conjecture 1.4 (Selmer). If $h \geq k \geq 3$, then the problem of finding $g_{\tilde{h}_{0}=h}(k)$ is uniquely solved by

$$
\mu_{i}= \begin{cases}\left\lfloor\frac{i(h-1)}{k-1}\right\rfloor-\left\lfloor\frac{(i-1)(h-1)}{k-1}\right\rfloor & \text { for } i=1, \ldots, k-2, \\ \left\lceil\frac{h-1}{k-1}\right\rceil & \text { for } i=k-1, \\ 1 & \text { for } i=k,\end{cases}
$$

in connection with (1.12) and (1.3). 
Remark 1.5.

- If $h<k$, then the problem is solved by Theorem 1.3. This follows from (1.7) because then $\mu_{k}^{(1)}=\mu_{k}^{(2)}=1$.

- If $h=k$, then (1.13) gives $\mu_{i}=1$ for $i=1, \ldots, k$. Then also $\mu_{i}^{(1)}=1$ for $i=1, \ldots, k$ while $\mu_{k}^{(2)}=2$. It follows that Conjecture 1.4 is true in the case $h=k$. We note that in this case $a_{i}=F_{2 i}$ for $i=1, \ldots, k$, where the $F_{2 i}$ are Fibonacci numbers, defined by $F_{1}=F_{2}=1, F_{n+2}=F_{n+1}+F_{n}$, $n=1,2, \ldots$

- If $h>k$, then $\mu_{k}^{(1)}>1$ and $\mu_{k}^{(2)}>1$, so that, by (1.7), we have $h>\widetilde{h}_{0}$ for both $h$-bases coming from Theorem 1.3. These bases, therefore, do not solve Selmer's problem.

In this paper we prove Selmer's Conjecture 1.4. The proof is based on Mrose's thesis [4]. In Section 2 we introduce certain determinants and indicate how the problem can be reformulated. The proof is then carried out in two steps, in Sections 3 and 4. Finally, some asymptotic estimates are given in Section 5.

2. Continuants and reformulation of the conjecture. The following definitions and results are taken from [4].

Definition 2.1. If $\left(x_{1}, \ldots, x_{k}\right)$ is a sequence of real numbers, then by a continuant $C\left(x_{1}, \ldots, x_{k}\right)$ we mean the determinant

$$
C\left(x_{1}, \ldots, x_{k}\right)=\left|\begin{array}{ccccccr}
x_{1} & -1 & 0 & 0 & \ldots & 0 & 0 \\
-1 & x_{2} & -1 & 0 & \ldots & 0 & 0 \\
0 & -1 & x_{3} & -1 & \ldots & 0 & 0 \\
\ldots \ldots & \ldots & \ldots & \ldots \ldots & \ldots & \ldots \ldots \\
0 & 0 & 0 & 0 & \ldots & -1 & x_{k}
\end{array}\right| .
$$

We also define

$$
C\left(x_{r}, \ldots, x_{s}\right)= \begin{cases}1 & \text { when } s=r-1 \\ 0 & \text { when } s \leq r-2 .\end{cases}
$$

The following result $[4$, p. 2] follows immediately.

Lemma 2.2. For $k \geq 1$ and real numbers $x_{1}, \ldots, x_{k}$ we have

$$
\begin{aligned}
C\left(x_{1}, \ldots, x_{k}\right) & =x_{1} C\left(x_{2}, \ldots, x_{k}\right)-C\left(x_{3}, \ldots, x_{k}\right) \\
& =x_{k} C\left(x_{1}, \ldots, x_{k-1}\right)-C\left(x_{1}, \ldots, x_{k-2}\right), \\
C\left(x_{1}, \ldots, x_{k}\right) & =C\left(x_{k}, \ldots, x_{1}\right) .
\end{aligned}
$$

Remark 2.3. In what follows, the elements $x_{1}, \ldots, x_{k}$ of a continuant $C\left(x_{1}, \ldots, x_{k}\right)$ will always be integers $\geq 2$. 
Re mark 2.4. The continuants are connected with continued fractions. If we denote by

$$
\left\langle a_{0}, a_{1}, a_{2}, \ldots\right\rangle=a_{0}-\frac{1}{a_{1}-\frac{1}{a_{2}-} \cdot}
$$

a reduced-regular ("reduziert-regelmässig" [5, p. 163]) continued fraction, then (see $[4$, p. 1$]$ and also $[5$, p. 8$]$ )

$$
\left\langle a_{0}, a_{1}, \ldots, a_{n}\right\rangle=\frac{C\left(a_{0}, a_{1}, \ldots, a_{n}\right)}{C\left(a_{1}, \ldots, a_{n}\right)} .
$$

Definition 2.5. A continuant $C\left(x_{1}, \ldots, x_{k}\right)$ with $x_{i}$ integers $\geq 2$ is called extremal if for any continuant $C\left(y_{1}, \ldots, y_{k}\right)$ with $y_{i}$ integers $\geq 2$ and $\sum_{i=1}^{k} x_{i}=\sum_{i=1}^{k} y_{i}$, we have $C\left(y_{1}, \ldots, y_{k}\right) \leq C\left(x_{1}, \ldots, x_{k}\right)$.

Definition 2.6. A sequence $\left(x_{1}, \ldots, x_{k}\right)$ of positive integers is called homogeneous if the following conditions are satisfied.

(a) There exists a positive integer $x$ such that $x_{i} \in\{x, x+1\}$ for $i=1, \ldots, k$.

$\left(\mathrm{b}_{1}\right)$ If $x_{i}=x$ and $x_{i+1}=x+1$ and if there exists a positive integer $t$ such that $x_{i-t} \neq x_{i+1+t}$, then for the smallest such $t$ we have $x_{i-t}=x+1$ and $x_{i+1+t}=x$.

$\left(\mathrm{b}_{2}\right)$ If there is no such $t$, then $i \leq k / 2$.

$\left(\mathrm{c}_{1}\right)$ If $x_{i}=x+1$ and $x_{i+1}=x$ and if there exists a positive integer $t$ such that $x_{i-t} \neq x_{i+1+t}$, then for the smallest such $t$ we have $x_{i-t}=x$ and $x_{i+1+t}=x+1$.

$\left(\mathrm{c}_{2}\right)$ If there is no such $t$, then $i \geq k / 2$.

Remark 2.7. The condition (c) above is equivalent with the requirement that the sequence $\left(x_{k}, \ldots, x_{1}\right)$ satisfies (b). A sequence $\left(x_{1}, \ldots, x_{k}\right)$ is therefore homogeneous if and only if $\left(x_{k}, \ldots, x_{1}\right)$ is homogeneous.

The following remarkable result [4, pp. 33 and 44] plays a key role in the proof of Theorem 1.3 and occupies a central position also in this paper.

Theorem 2.8 (Mrose). Let $k \geq 1$ and $s \geq 2 k$ be given positive integers. In case $\operatorname{gcd}(k, s+1)=1$ there is exactly one homogeneous sequence $\left(x_{1}, \ldots, x_{k}\right)$ and extremal continuant $C\left(x_{1}, \ldots, x_{k}\right)$ with $\sum_{i=1}^{k} x_{i}=s$. In case $\operatorname{gcd}(k, s+1)>1$ there are exactly two homogeneous sequences $\left(x_{1}, \ldots, x_{k}\right)$ and $\left(y_{1}, \ldots, y_{k}\right)$ and extremal continuants $C\left(x_{1}, \ldots, x_{k}\right)$ and $C\left(y_{1}, \ldots, y_{k}\right)$ with $\sum_{i=1}^{k} x_{i}=\sum_{i=1}^{k} y_{i}=s$. Let $x=\lfloor s / k\rfloor, m=s-\lfloor s / k\rfloor k$, and $n=k-m=k\lceil(s+1) / k\rceil-s$. The sequences $\left(x_{1}, \ldots, x_{k}\right)$ and $\left(y_{1}, \ldots, y_{k}\right)$ 
are given by

$$
\begin{aligned}
x_{i}= \begin{cases}\left\lfloor\frac{i(s+1)}{k}\right\rfloor-\left\lfloor\frac{(i-1)(s+1)}{k}\right\rfloor & \text { for } i=1, \ldots, k-1, \\
s-\left\lfloor\frac{(k-1)(s+1)}{k}\right\rfloor & \text { for } i=k,\end{cases} \\
= \begin{cases}x \quad \text { for } i=\left\lceil\frac{(r-1) k+1}{n-1}\right\rceil & (r=1, \ldots, n-1) \text { and } i=k, \\
x+1 \quad \text { for } i=\left\lceil\frac{k r}{m+1}\right\rceil(r=1, \ldots, m),\end{cases} \\
y_{i}= \begin{cases}\left\lceil\frac{s+1}{k}\right\rceil-1 & \text { for } i=1, \\
\left\lceil\frac{i(s+1)}{k}\right\rceil-\left\lceil\frac{(i-1)(s+1)}{k}\right\rceil=2, \ldots, k,\end{cases} \\
=\left\{\begin{array}{l}
x \quad \text { for } i=1 \text { and } i=\left\lceil\frac{k(r-1)}{n-1}\right\rceil(r=2, \ldots, n), \\
x+1 \quad \text { for } i=\left\lceil\frac{k r+1}{m+1}\right\rceil(r=1, \ldots, m) .
\end{array}\right.
\end{aligned}
$$

The sequences $\left(x_{1}, \ldots, x_{k}\right)$ and $\left(y_{1}, \ldots, y_{k}\right)$ satisfy the conditions

$$
x_{k+1-i}=y_{i} \quad \text { for } i=1, \ldots, k,
$$

$$
\left(x_{1}, \ldots, x_{k}\right)=\left(y_{1}, \ldots, y_{k}\right) \text { if and only if } \operatorname{gcd}(k, s+1)=1 .
$$

Using Theorem 1.2 and other results of Hofmeister [1], Mrose [4, p. 68] proved

Theorem 2.9 (Mrose). If $h \geq 1$ and $k \geq 1$ are given positive integers, then $A_{k}=\left\{a_{1}, \ldots, a_{k}\right\}$ is an extremal regular $h$-basis $\widetilde{A}_{k}^{*}$ if and only if there exists a sequence $\left(x_{1}, \ldots, x_{k}\right)$ of integers $\geq 2$ having the following properties.

(a)

$$
a_{i}=C\left(x_{1}, \ldots, x_{i-1}\right) \quad \text { for } i=1, \ldots, k,
$$

(b)

$$
\sum_{i=1}^{k} x_{i}=h+2 k-1,
$$

(c) the continuant $C\left(x_{1}, \ldots, x_{k}\right)$ is extremal.

If these conditions are satisfied, then

$$
g_{h}(k)=g_{h}\left(\widetilde{A}_{k}^{*}\right)=C\left(x_{1}, \ldots, x_{k}\right)-1 .
$$


Re mark 2.10. Given a basis $A_{k}=\left\{a_{1}, \ldots, a_{k}\right\}$, Mrose [4, p. 49] defined recursively

$$
m_{j}=\left\lfloor\frac{a_{j+1}-\sum_{i=2}^{j-1} m_{i} a_{i}}{a_{j}}\right\rfloor \quad \text { for } j=1, \ldots, k-1 .
$$

Comparing (2.10) with (1.4), we easily deduce that $\mu_{1}=m_{1}-2, \mu_{2}=m_{2}-1$, and $\mu_{i}=m_{i}$ for $i=3, \ldots, k-1$. From this it follows [4, p. 59] that for the sequence $\left(x_{1}, \ldots, x_{k}\right)$ of Theorem 2.9 we have

$$
\begin{aligned}
& x_{i}=\mu_{i}+2 \quad \text { for } i=1, \ldots, k-1, \\
& x_{k}=\mu_{k}+1 .
\end{aligned}
$$

In preparation of our reformulation of Conjecture 1.4, we now briefly sketch the proof of Theorem 1.3. If we use Theorem 2.8 with $s=h+2 k-1$ and associate, by (2.11) and (2.12), the $\mu_{i}^{(1)}$ with the $x_{i}$ and the $\mu_{i}^{(2)}$ with the $y_{i}$, we easily get (1.10) and (1.11). Using (2.11) and (2.1), (1.12) follows from (2.7). Finally, (1.3) follows easily by induction from (2.9), using (2.11) and (2.12). We have thus seen how Theorem 1.3 follows from Theorems 2.8 and 2.9 .

We now turn our attention to Conjecture 1.4. We find it convenient to introduce the following definitions (not to be found in [4]).

Definition 2.11. We call the sequences $\left(x_{1}, \ldots, x_{k}\right)$ and $\left(y_{1}, \ldots, y_{k}\right)$ of Theorem 2.8 Mrose's first and second sequence (for s), respectively.

DeFinition 2.12. We call a continuant $C\left(x_{1}, \ldots, x_{k-1}, 2\right)$ with $x_{i}$ integers $\geq 2$ a 2-extremal continuant if for every continuant $C\left(y_{1}, \ldots, y_{k-1}, 2\right)$ with $y_{i}$ integers $\geq 2$ and $\sum_{i=1}^{k-1} x_{i}=\sum_{i=1}^{k-1} y_{i}$ we have

$$
C\left(y_{1}, \ldots, y_{k-1}, 2\right) \leq C\left(x_{1}, \ldots, x_{k-1}, 2\right) .
$$

It follows from Theorem 2.9, (1.7), and (2.12) that the problem behind Conjecture 1.4 can be reformulated in the following way.

Problem 2.13. If $h \geq k \geq 3$, find 2-extremal continuants $C\left(x_{1}, \ldots\right.$, $\left.x_{k-1}, 2\right)$ such that $\sum_{i=1}^{k-1} x_{i}=h+2 k-3$ (and then use (2.7) and (2.9)).

Comparing (1.13) with (1.10), and taking the above sketch of the proof of Theorem 1.3 into consideration, we see that Conjecture 1.4 is equivalent with the following result.

THEOREM 2.14. Let $h \geq k \geq 3$. There is exactly one 2-extremal continuant $C\left(x_{1}, \ldots, x_{k-1}, 2\right)$ with $\sum_{i=1}^{k-1} x_{i}=h+2 k-3$, and it satisfies the condition that the sequence $\left(x_{1}, \ldots, x_{k-2}, x_{k-1}-1\right)$ is Mrose's first sequence (2.3) $($ for $s=h+2 k-4)$.

Theorem 2.14, that is, Selmer's Conjecture 1.4, will be proved in two steps, as Theorems 2.15 and 2.16 below. 
Theorem 2.15. Let $h \geq k \geq 3$. If $C\left(x_{1}, \ldots, x_{k-1}, 2\right)$ is a 2-extremal continuant with $\sum_{i=1}^{k-1} x_{i}=h+2 k-3$, then the sequence $\left(x_{1}, \ldots, x_{k-2}\right.$, $\left.x_{k-1}-1\right)$ is homogeneous.

This first step leaves us, according to Theorem 2.8, at most two possibilities for a 2-extremal continuant of the desired kind. The second step is then

TheOREM 2.16. Let $k \geq 3$. If $\left(x_{1}, \ldots, x_{k-1}\right)$ and $\left(y_{1}, \ldots, y_{k-1}\right)$ are Mrose's first and second sequence, respectively (for $s \geq 2(k-1))$, then, if $\left(x_{1}, \ldots, x_{k-1}\right) \neq\left(y_{1}, \ldots, y_{k-1}\right)$, we have

$$
C\left(x_{1}, \ldots, x_{k-2}, x_{k-1}+1,2\right)>C\left(y_{1}, \ldots, y_{k-2}, y_{k-1}+1,2\right) .
$$

Example 2.17. Let $h=15, k=9$. Calculating the coefficients $\mu_{i}$ of Conjecture 1.4 we get

$\mu_{1}=1, \mu_{2}=2, \mu_{3}=2, \mu_{4}=2, \mu_{5}=1, \mu_{6}=2, \mu_{7}=2, \mu_{8}=2, \mu_{9}=1$. Using (1.10) and (1.3) we get the regular $h$-basis

$$
A_{k}=\{1,3,11,41,153,418,1519,5658,21113\}
$$

and the regular $h$-range

$$
g_{h}\left(A_{k}\right)=36567 .
$$

Now $s=h+2 k-4=29$ and denoting by $\left(x_{1}, \ldots, x_{8}\right)$ and $\left(y_{1}, \ldots, y_{8}\right)$ Mrose's first and second sequence, respectively (for $s=29$ ), we find

$$
\begin{aligned}
\left(x_{1}, \ldots, x_{8}\right) & =(3,4,4,4,3,4,4,3), \\
\left(y_{1}, \ldots, y_{8}\right) & =(3,4,4,3,4,4,4,3) .
\end{aligned}
$$

Using (2.7) with the sequence $\left(x_{1}, \ldots, x_{7}, x_{8}+1,2\right)=(3,4,4,4,3,4,4,4,2)$ we get (2.13) and using (2.9) we get (2.14), since $C(3,4,4,4,3,4,4,4,2)=$ 36568. Note that

$$
C\left(y_{1}, \ldots, y_{7}, y_{8}+1,2\right)=C(3,4,4,3,4,4,4,4,2)=36567,
$$

illustrating Theorem 2.16 .

3. First step: proof of Theorem 2.15. The first step is taken on a well-trodden path, since we can take as a model Mrose's Satz 3.1 [4, p. 19] (which says that an extremal continuant is homogeneous).

Re mark 3.1. Leaving now [7] and staying with [4] for the rest of this paper, we find it convenient to make a slight change in the notation: the letter $k$, which from now on will denote an integer $\geq 2$, will lose its former meaning.

Theorem 2.15 will be proved in the following form. Let

$$
h \geq k+1
$$


and let $C\left(x_{1}, \ldots, x_{k}, 2\right)$ be a 2 -extremal continuant with

$$
\sum_{i=1}^{k} x_{i}=h+2 k-1 .
$$

Then

$$
\left(x_{1}, \ldots, x_{k-1}, x_{k}-1\right) \text { is homogeneous. }
$$

The proof of (3.3) will be preceded by a series of lemmas. Before starting with the proofs, we introduce the following

Definition 3.2. If a sequence $\left(x_{1}, \ldots, x_{k}\right)$ satisfies the conditions (a), $\left(b_{1}\right)$, and $\left(c_{1}\right)$ of Definition 2.6, we say that it is weakly homogeneous.

Lemma 3.3. Suppose that $C\left(x_{1}, \ldots, x_{k}, 2\right)$ is a 2-extremal continuant. Then the sequence $\left(x_{1}, \ldots, x_{k}\right)$ is weakly homogeneous.

Proof. The proof is similar to the proof of Satz 3.1 in $[4$, p. 19] (see Remark 3.4 below).

Remark 3.4. Mrose was able to make certain short cuts, using the symmetry of the situation (our (2.2) and Remark 2.7). Note that while the analogue of Remark 2.7 clearly holds for weakly homogeneous sequences, we do not have the full symmetry of the situation here, caused by the last element 2 of the continuant. We therefore define, for $1 \leq p<q \leq k$,

$$
y_{i}= \begin{cases}x_{i} & \text { if } i \neq p, q \\ x_{p}-1 & \text { if } i=p \\ x_{q}+1 & \text { if } i=q .\end{cases}
$$

Then we have, analogously to $(9)$ in $[4$, p. 20],

$$
\begin{aligned}
C & \left(x_{1}, \ldots, x_{k}, 2\right)-C\left(y_{1}, \ldots, y_{k}, 2\right) \\
= & \left(1-x_{p}+x_{q}\right) C\left(x_{1}, \ldots, x_{p-1}\right) C\left(x_{p+1}, \ldots, x_{q-1}\right) C\left(x_{q+1}, \ldots, x_{k}, 2\right) \\
& -C\left(x_{1}, \ldots, x_{p-1}\right) C\left(x_{p+1}, \ldots, x_{q-2}\right) C\left(x_{q+1}, \ldots, x_{k}, 2\right) \\
& -C\left(x_{1}, \ldots, x_{p-1}\right) C\left(x_{p+1}, \ldots, x_{q-1}\right) C\left(x_{q+2}, \ldots, x_{k}, 2\right) \\
& +C\left(x_{1}, \ldots, x_{p-2}\right) C\left(x_{p+1}, \ldots, x_{q-1}\right) C\left(x_{q+1}, \ldots, x_{k}, 2\right) \\
& +C\left(x_{1}, \ldots, x_{p-1}\right) C\left(x_{p+2}, \ldots, x_{q-1}\right) C\left(x_{q+1}, \ldots, x_{k}, 2\right) .
\end{aligned}
$$

Lemma 3.5. If $C\left(x_{1}, \ldots, x_{k}, 2\right)$ is a 2-extremal continuant satisfying (3.1) and (3.2), then $x_{i} \geq 3$ for $i=1, \ldots, k$.

Proof. According to Lemma 3.3, we have, for some integer $x, x_{i} \in$ $\{x, x+1\}$ for $i=1, \ldots, k$. If, for some $j \in\{1, \ldots, k\}$, we have $x_{j}=2$, then $x=2$ and so $h+2 k-1=\sum_{i=1}^{k} x_{i}<3 k$, contradicting (3.1). 
LEMmA 3.6. If $x_{1}, \ldots, x_{j}$ are any integers $\geq 2$ and $x_{j} \geq 3$, then

$$
C\left(x_{1}, \ldots, x_{j}\right)>2 C\left(x_{1}, \ldots, x_{j-1}\right) .
$$

Proof. Let $x_{j}=2+t_{j}$, where $t_{j} \geq 1$. From $(2.1)$ and Satz $1.7[4$, p. 7$]$ we get

$$
\begin{aligned}
C\left(x_{1}, \ldots, x_{j}\right)-2 C\left(x_{1}, \ldots, x_{j-1}\right) & \\
& =t_{j} C\left(x_{1}, \ldots, x_{j-1}\right)-C\left(x_{1}, \ldots, x_{j-2}\right) \\
& \geq C\left(x_{1}, \ldots, x_{j-1}\right)-C\left(x_{1}, \ldots, x_{j-2}\right)>0 .
\end{aligned}
$$

Lemma 3.7. Let $C\left(x_{1}, \ldots, x_{k}, 2\right)$ be a 2-extremal continuant satisfying, for some integer $x \geq 3$, the condition $x_{i} \in\{x, x+1\}$ for $i=1, \ldots, k$. If, for some $j \in\{1, \ldots, k\}$, we have $x_{j}=x+1$, then $x_{k}=x+1$.

Proof. Suppose that $x_{k}=x$. Let $p$ be the greatest number such that $x_{p}=x+1$. We let $q=k$ and define a sequence $\left(y_{1}, \ldots, y_{k}\right)$ by (3.4). Now $1-x_{p}+x_{q}=0$ and so (3.5) gives

$$
\begin{aligned}
C\left(x_{1}, \ldots, x_{k}, 2\right)-C & \left(y_{1}, \ldots, y_{k}, 2\right) \\
= & -C\left(x_{1}, \ldots, x_{p-1}\right) C\left(x_{p+1}, \ldots, x_{q-2}\right) C(2) \\
& -C\left(x_{1}, \ldots, x_{p-1}\right) C\left(x_{p+1}, \ldots, x_{q-1}\right) \cdot 1 \\
& +C\left(x_{1}, \ldots, x_{p-2}\right) C\left(x_{p+1}, \ldots, x_{q-1}\right) C(2) \\
& +C\left(x_{1}, \ldots, x_{p-1}\right) C\left(x_{p+2}, \ldots, x_{q-1}\right) C(2) .
\end{aligned}
$$

By the definition of $p$, if $p<i<q$, then $x_{i}=x$. It follows that $C\left(x_{p+1}, \ldots\right.$, $\left.x_{q-2}\right)=C\left(x_{p+2}, \ldots, x_{q-1}\right)$, so that (3.7) and (3.6) give

$$
\begin{aligned}
& C\left(x_{1}, \ldots, x_{k}, 2\right)-C\left(y_{1}, \ldots, y_{k}, 2\right) \\
& \quad=C\left(x_{p+1}, \ldots, x_{q-1}\right)\left(2 C\left(x_{1}, \ldots, x_{p-2}\right)-C\left(x_{1}, \ldots, x_{p-1}\right)\right)<0,
\end{aligned}
$$

which contradicts the fact that $C\left(x_{1}, \ldots, x_{k}, 2\right)$ is a 2 -extremal continuant.

Proof of (3.3). We suppose that $C\left(x_{1}, \ldots, x_{k}, 2\right)$ is a 2-extremal continuant satisfying (3.1) and (3.2). We have to prove that the sequence $\left(x_{1}, \ldots, x_{k-1}, x_{k}-1\right)$ satisfies the conditions $(\mathrm{a}),\left(\mathrm{b}_{1}\right),\left(\mathrm{b}_{2}\right),\left(\mathrm{c}_{1}\right)$, and $\left(\mathrm{c}_{2}\right)$ of Definition 2.6.

(a) It follows immediately from Lemmas $3.3,3.5$, and 3.7 that $\left(x_{1}, \ldots\right.$, $\left.x_{k-1}, x_{k}-1\right)$ satisfies (a).

If $x_{i}=x$ for $i=1, \ldots, k$, then the sequence $\left(x_{1}, \ldots, x_{k-1}, x_{k}-1\right)$ is clearly homogeneous. For the rest of the proof, we may therefore suppose that for an integer $x \geq 3$, we have $x_{i} \in\{x, x+1\}$ for $i=1, \ldots, k$ and that

$$
x_{k}-1=x .
$$

Moreover, since the sequence $(x, x, \ldots, x)$ is clearly homogeneous, we may suppose that for some $j \in\{1, \ldots, k-1\}, x_{j}=x+1$. 
$\left(b_{1}\right)$ We know from Lemma 3.3 that $\left(x_{1}, \ldots, x_{k}\right)$ satisfies $\left(b_{1}\right)$. It follows immediately from (3.8) that also $\left(x_{1}, \ldots, x_{k-1}, x_{k}-1\right)$ satisfies $\left(b_{1}\right)$.

$\left(\mathrm{b}_{2}\right)$ Suppose that $\left(\mathrm{b}_{2}\right)$ fails. Then there exists an integer $r, k / 2<r<$ $k-1$, such that $x_{r}=x, x_{r+1}=x+1, x_{r-i}=x_{r+1+i}$ for $i=1, \ldots, k-r-2$, and

$$
x_{2 r-k+1}=x_{k}-1 \text {. }
$$

We define a sequence $\left(y_{1}, \ldots, y_{k}\right)$ by (8) in $[4$, p. 19] with $p=r, q=r+1$. Using (10) in [4, p. 23], (3.9), and (3.8), we obtain

$$
\begin{aligned}
C\left(x_{1}, \ldots, x_{k}, 2\right)- & C\left(y_{1}, \ldots, y_{k}, 2\right) \\
= & 2 C\left(x_{1}, \ldots, x_{2 r-k+1}\right)-C\left(x_{1}, \ldots, x_{2 r-k}\right) C\left(x_{k}, 2\right) \\
& =-2 C\left(x_{1}, \ldots, x_{2 r-k-1}\right)-C\left(x_{1}, \ldots, x_{2 r-k}\right)<0,
\end{aligned}
$$

a contradiction. This proves $\left(\mathrm{b}_{2}\right)$.

$\left(c_{1}\right)$ and $\left(c_{2}\right)$ can be proved in a similar manner, using (3.4).

We have proved Theorem 2.15.

4. Second step: proof of Theorem 2.16. In this section we take a closer look at Mrose's sequences (2.3) and (2.4). First of all, we have to consider the following question: If Mrose's two sequences are different, how do they differ from each other?

Let $\left(x_{1}, \ldots, x_{k}\right)$ and $\left(y_{1}, \ldots, y_{k}\right)$ be Mrose's first and second sequence, respectively (for $s \geq 2 k$ ), and let $x=\lfloor s / k\rfloor$. Theorem 2.8 implies

Lemma 4.1 (Mrose). (a) If $s \equiv-1(\bmod k)$, we have

$$
\begin{aligned}
\left(x_{1}, \ldots, x_{k}\right) & =(x+1, x+1, \ldots, x+1, x), \\
\left(y_{1}, \ldots, y_{k}\right) & =(x, x+1, x+1, \ldots, x+1) .
\end{aligned}
$$

(b) If $s \not \equiv-1(\bmod k)$, we have

$$
x_{1}=y_{1}=x_{k}=y_{k}=x .
$$

LEMma 4.2. We have

$$
\begin{aligned}
C\left(x_{1}, \ldots, x_{k-1}, x_{k}+1,2\right)-C\left(y_{1}, \ldots, y_{k-1}, y_{k}+1,2\right) \\
=C\left(x_{1}, \ldots, x_{k-1}, x_{k}+1\right)-C\left(y_{1}, \ldots, y_{k-1}, y_{k}+1\right) \\
=C\left(x_{1}, \ldots, x_{k-1}\right)-C\left(y_{1}, \ldots, y_{k-1}\right) .
\end{aligned}
$$

Pr o of. This can easily be proved using (2.1), (2.2), (2.5), and Lemma 4.1 .

Using Lemma 4.2 (and keeping Remark 3.1 in mind), we prove Theorem 2.16 in the following form. If $\left(x_{1}, \ldots, x_{k}\right)$ and $\left(y_{1}, \ldots, y_{k}\right)$ are Mrose's first 
and second sequence, respectively (for $s \geq 2 k$ ), then

$$
C\left(x_{1}, \ldots, x_{k-1}\right)>C\left(y_{1}, \ldots, y_{k-1}\right) .
$$

If $s \equiv-1(\bmod k)$, then we see immediately from Lemma 4.1 that (4.3) holds. (Alternatively, we could use Lemma 3.3 and note that as the sequence $\left(y_{1}, \ldots, y_{k-1}, y_{k}+1\right)$ does not satisfy the condition (a) of Definition 2.6, then $C\left(y_{1}, \ldots, y_{k-1}, y_{k}+1,2\right)$ is not a 2 -extremal continuant.) From now on, we will therefore suppose that

$$
s \not \equiv-1(\bmod k) .
$$

The following result provides a fundamental tool for our investigations.

LEMmA 4.3. Let $s \geq 2 k$, assume (4.4), and let $\left(x_{1}, \ldots, x_{k}\right)$ and $\left(y_{1}, \ldots, y_{k}\right)$ be Mrose's first and second sequence, respectively. Let $\operatorname{gcd}(k, s+$ $1)=d$ and $x=\lfloor s / k\rfloor$. Consider the box

$$
\begin{aligned}
& x_{1}, \ldots, x_{k} \\
& y_{1}, \ldots, y_{k}
\end{aligned}
$$

This box has the following form.

(a) At both ends of the box (4.5) there are subboxes of the form

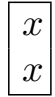

(b) There are $d-1$ subboxes

$$
\begin{array}{|cc|}
\hline x+1 & x \\
x & x+1 \\
\hline
\end{array}
$$

where the corresponding elements of $\left(x_{1}, \ldots, x_{k}\right)$ and $\left(y_{1}, \ldots, y_{k}\right)$ differ from each other.

(c) The boxes in (a) and (b) are separated by (possibly empty) identical, symmetrical subboxes with the same corresponding elements.

EXAmple 4.4. We take $k=21, s=74$ (see [4, p. 71]) to illustrate the situation. In this case $d=\operatorname{gcd}(21,75)=3$ and (4.5) is

\begin{tabular}{|l|lllll|ll|lllll|ll|lllll|l|}
\hline 3 & 4 & 3 & 4 & 3 & 4 & 4 & 3 & 4 & 3 & 4 & 3 & 4 & 4 & 3 & 4 & 3 & 4 & 3 & 4 & 3 \\
3 & 4 & 3 & 4 & 3 & 4 & 3 & 4 & 4 & 3 & 4 & 3 & 4 & 3 & 4 & 4 & 3 & 4 & 3 & 4 & 3 \\
\hline
\end{tabular}

The following proof was suggested by Veikko Ennola. It has the merit of being simpler and shorter than the author's original proof, which proceeded by induction on $d$.

Proof of Lemma 4.3. We note that (a) follows immediately from (4.2). To prove (b) and (c), we use (2.3) and (2.4) in connection with the 
obvious formula

$$
\lceil\xi\rceil-\lfloor\xi\rfloor= \begin{cases}1 & \text { if } \xi \notin \mathbb{Z}, \\ 0 & \text { if } \xi \in \mathbb{Z} .\end{cases}
$$

We write $s+1=d a$ and $k=d b$, so that $\operatorname{gcd}(a, b)=1$ and, by (4.4), $b>1$. If $1<i<k$, then we have

$$
x_{i}-y_{i}=\left\lfloor\frac{i a}{b}\right\rfloor-\left\lceil\frac{i a}{b}\right\rceil-\left\lfloor\frac{(i-1) a}{b}\right\rfloor+\left\lceil\frac{(i-1) a}{b}\right\rceil,
$$

so that, by (4.7),

$$
x_{i}-y_{i}= \begin{cases}0 & \text { if } i \not \equiv 0,1(\bmod b) \\ 1 & \text { if } i \equiv 0(\bmod b) \\ -1 & \text { if } i \equiv 1(\bmod b) .\end{cases}
$$

In a similar fashion,

$$
\begin{aligned}
x_{i}-x_{i+b} & =\left\lfloor\frac{i a}{b}\right\rfloor-\left\lfloor\frac{(i-1) a}{b}\right\rfloor-\left\lfloor\frac{(i+b) a}{b}\right\rfloor+\left\lfloor\frac{(i+b-1) a}{b}\right\rfloor \\
& =0 \text { if } i \not \equiv 0,1(\bmod b), \\
x_{i}-x_{b+1-i} & =\left\lfloor\frac{i a}{b}\right\rfloor-\left\lceil\frac{i a}{b}\right\rfloor+\left\lceil\frac{(i-1) a}{b}\right\rfloor-\left\lfloor\frac{(i-1) a}{b}\right\rfloor \\
& =0 \text { if } i \not \equiv 0,1(\bmod b) .
\end{aligned}
$$

(b) and (c) now follow immediately from (4.8)-(4.10).

We introduce the following notation.

Definition 4.5. If $\left(x_{1}, \ldots, x_{j}\right)$ and $\left(y_{1}, \ldots, y_{j}\right)$ are any sequences with $x_{i}, y_{i}$ integers $\geq 2$ for $i=1, \ldots, j$, we write

$$
C\left(x_{1}, \ldots, x_{j}\right)=u_{j}, \quad C\left(y_{1}, \ldots, y_{j}\right)=v_{j}, \quad d_{j}=u_{j}-v_{j} .
$$

According to this notation, if $\left(x_{1}, \ldots, x_{k}\right)$ and $\left(y_{1}, \ldots, y_{k}\right)$ are Mrose's first and second sequence, respectively, then (4.3) can be stated as

$$
d_{k-1}>0 .
$$

We find it convenient to prove a little more than (4.12). In fact, if $t \geq 0$ is the length of the symmetrical subbox in Lemma 4.3 , and if $\left(x_{1}, \ldots, x_{k}\right) \neq$ $\left(y_{1}, \ldots, y_{k}\right)$, we show that

$$
\begin{gathered}
d_{1}=\ldots=d_{t+1}=0, \\
d_{t+2}>0, \ldots, d_{k-1}>0, \quad \text { and } \\
d_{k}=0 .
\end{gathered}
$$

Here (4.13) is clear from Lemma 4.3, and (4.15) follows from (2.5) and (2.2). 

that

Remark 4.6. If $s \equiv-1(\bmod k)$, then it follows immediately from (4.1)

$$
d_{1}>0, \ldots, d_{k-1}>0, \quad d_{k}=0 .
$$

Our goal now is to prove (4.14). The proof is based on Lemma 4.3.

R e mark 4.7. Actually, we get the result for a little wider class of pairs of sequences than just for Mrose's sequences, since from the elements of the symmetrical subboxes we need, indeed, only the fact that they form a symmetrical sequence. Their actual values do not concern us. In particular, they do not have to be in the set $\{x, x+1\}$.

LEMMA 4.8. Let

$$
\begin{aligned}
& \left(x_{1}, \ldots, x_{k}\right)=\left(z^{*}, z_{1}, \ldots, z_{t}, z+1, z, z_{t}, \ldots, z_{1}\right), \\
& \left(y_{1}, \ldots, y_{k}\right)=\left(z^{*}, z_{1}, \ldots, z_{t}, z, z+1, z_{t}, \ldots, z_{1}\right),
\end{aligned}
$$

with $t \geq 0, z^{*}, z, z_{i}$ integers $\geq 2$ for $i=1, \ldots, t$. Then

$$
\begin{gathered}
d_{i}=0 \quad \text { for } i=1, \ldots, t+1, \quad \text { and } \\
d_{k-i}=u_{i} \quad \text { for } i=0, \ldots, t+1 .
\end{gathered}
$$

Proof. (4.17) is trivial. Note that, by Definitions 2.1 and $4.5, u_{0}=1$. It follows immediately from (2.1) that

$$
d_{i}=x_{i} u_{i-1}-y_{i} v_{i-1}-d_{i-2} \quad \text { for } i=1, \ldots, k
$$

(with $d_{0}=d_{-1}=0$ ). Using (4.19) and (4.17) we easily obtain

$$
d_{t+2}=u_{t+1} \quad \text { and } \quad d_{t+3}=u_{t} .
$$

We now prove (4.18) in the form

$$
d_{t+2+i}=u_{t+1-i} \quad \text { for } i=0, \ldots, t+1,
$$

using induction on $i$.

1. If $i=0$ or $i=1,(4.21)$ follows from (4.20).

2. The general induction step (for $t>0$ ) follows easily from the obvious formulas

$$
\begin{gathered}
u_{i}=z_{i-1} u_{i-1}-u_{i-2} \quad \text { for } i=2, \ldots, t+1, \\
d_{t+2+i}=z_{t-i+2} d_{t+i+1}-d_{t+i} \quad \text { for } i=2, \ldots, t+1 .
\end{gathered}
$$

Lemma 4.9. Let $x, x_{i}$ be integers $\geq 2$ for $i=1, \ldots, n$. Let

$$
b_{r}=C\left(x_{1}, \ldots, x_{n}, x, x_{1}, \ldots, x_{n}, x, \ldots, x, x_{1}, \ldots, x_{n}\right),
$$

where $r$ is the number of occurrences of the symbol $x$ in the continuant. Let $a=C\left(x_{1}, \ldots, x_{n}\right)$. Then $a \mid b_{r}$ for $r=0,1, \ldots$

Pr o of. Easy by induction on $r$ using Satz $1.2(2)$ in [4, p. 3]. 
LEMMA 4.10. Let

$$
\begin{aligned}
\left(x_{1}, \ldots, x_{k}\right) & =\left(x_{1}, \ldots, x_{n}, x+1, x\right), \\
\left(y_{1}, \ldots, y_{k}\right) & =\left(y_{1}, \ldots, y_{n}, x, x+1\right),
\end{aligned}
$$

where $x, x_{i}, y_{i}$ are integers $\geq 2$ for $i=1, \ldots, n$. If $d_{n-1}=x d_{n}$, then we have

$$
\begin{gathered}
d_{k-1}=u_{n}, \\
d_{k}=u_{n-1}-d_{n} .
\end{gathered}
$$

Pr o of. Easy calculation.

Our goal, (4.14), will finally be reached by the next result (see Remark 4.7).

Lemma 4.11. Let $t \geq 0$, let $x, z_{i}$ be integers $\geq 2$ for $i=1, \ldots, t$, and let $\left(z_{1}, \ldots, z_{t}\right)$ be a symmetrical sequence. Let

$$
\begin{gathered}
\left(x_{1}, \ldots, x_{k}\right)=\left(x, z_{1}, \ldots, z_{t}, x+1, x, z_{1}, \ldots, z_{t}, x+1,\right. \\
\left.x, \ldots, x+1, x, z_{1}, \ldots, z_{t}, x\right), \\
\left(y_{1}, \ldots, y_{k}\right)=\left(x, z_{1}, \ldots, z_{t}, x, x+1, z_{1}, \ldots, z_{t}, x\right. \\
\left.x+1, \ldots, x, x+1, z_{1}, \ldots, z_{t}, x\right)
\end{gathered}
$$

be two sequences such that their box (4.5) contains g subboxes (4.6). (If $\left(x_{1}, \ldots, x_{k}\right)$ and $\left(y_{1}, \ldots, y_{k}\right)$ are Mrose's first and second sequence, respectively $($ for $s \geq 2 k, s \not \equiv-1(\bmod k))$, then, by Lemma 4.3, $g=d-1$, where $d=\operatorname{gcd}(k, s+1)$.) Then $d_{1}=\ldots=d_{t+1}=0, d_{k}=d_{(g+1)(t+2)}=0$, and

$$
d_{j(t+2)+i}=r_{j} u_{t+1-i} \quad \text { for } j=1, \ldots, g, i=0, \ldots, t+1,
$$

where

$$
\begin{gathered}
r_{j}=\frac{u_{j(t+2)-1}}{u_{t+1}} \text { are integers, and } \\
1=r_{1}<\ldots<r_{g} .
\end{gathered}
$$

Pr o of. Again, the claim about the zero values of the $d_{i}$ 's is trivial. We note first that (4.25) follows from Lemma 4.9 and (4.26) follows from Satz 1.7 [4, p. 7]. We prove (4.24) by induction on $g$.

1. If $g=1$, then (4.24) follows from Lemma 4.8.

2. Suppose now that $g>1$. We may assume that (4.24) holds for $j=$ $1, \ldots, g-1, i=0, \ldots, t+1$. Since $u_{0}=1$ and $u_{1}=x$, it follows from our induction hypothesis, and formulas (4.22) and (4.23), that

$$
\begin{aligned}
d_{g(t+2)} & =u_{g(t+2)-1}, \\
d_{g(t+2)+1} & =u_{g(t+2)-2}-d_{g(t+2)-1} .
\end{aligned}
$$

We have to prove that 


$$
d_{g(t+2)+i}=r_{g} u_{t+1-i} \quad \text { for } i=0, \ldots, t+1 .
$$

We prove (4.29) by induction on $i$.

(A) If $i=0$, then (4.29) follows immediately from (4.27) and the definition of $r_{g}$.

If $i=1$, we use (4.28) and the induction hypothesis for $g$, and get

$$
d_{g(t+2)+1}=u_{(g-1)(t+2)+t}-\frac{u_{(g-1)(t+2)-1}}{u_{t+1}} .
$$

It follows that (4.29) holds for $i=1$ if we have

$$
u_{t+1} u_{(g-1)(t+2)+t}-u_{(g-1)(t+2)-1}=u_{g(t+2)-1} u_{t} .
$$

But if we apply Satz $1.3\left[4\right.$, p. 3] to the sequence $\left(x_{1}, \ldots, x_{k}\right)$, and use the symmetry of the subsequence $\left(z_{1}, \ldots, z_{t}\right)$, we get (4.30) without difficulty (taking $m=n-1=k-t-2, j=t+1$ in Satz 1.3). So (4.29) holds for $i=1$.

(B) If $t>0$ we continue easily by induction on $i$ in a similar manner to the proof of Lemma 4.8 .

This completes the proof of (4.24) by induction on $g$.

Our goal, (4.14), now follows immediately from (4.24) and (4.26) since, by Satz 1.7 [4, p. 7], the numbers $u_{j}$ are positive. (4.14) implies (4.12), which, in turn, implies (4.3). Theorem 2.16 is now proved. As Theorem 2.15 was proved in the previous section, we have proved Theorem 2.14. This means that we have proved Selmer's Conjecture.

Remark 4.12. (4.13), (4.14), and (4.16) give also information, using (2.7), about the size relations of the corresponding basis elements of the two extremal regular $h$-bases in Theorem 1.3 (in case the bases are different).

Remark 4.13. Keeping in mind our question from the beginning of this section, we note that if $\left(x_{1}, \ldots, x_{k}\right)$ and $\left(y_{1}, \ldots, y_{k}\right)$ are Mrose's first and second sequence, respectively (for $s \geq 2 k$ ), and if $\left(x_{1}, \ldots, x_{k}\right) \neq\left(y_{1}, \ldots, y_{k}\right)$, then Remark 2.4, (2.5), (2.2), and (4.3) imply that

$$
\left\langle x_{1}, \ldots, x_{k}\right\rangle>\left\langle y_{1}, \ldots, y_{k}\right\rangle .
$$

We conclude this section by giving an example of Lemma 4.11 (and Remark 4.12).

EXAMPle 4.14. We use the sequences of our earlier Example 4.4 (see [4, p. 71]). In this case we have $g=2, t=5, r_{1}=1, r_{2}=\frac{u_{13}}{u_{6}}=\frac{3909217}{1079}=$ 3623 . We give below a table of values of $u_{i}, v_{i}$, and $d_{i}$ for $i=1, \ldots, 21$. 
Table

\begin{tabular}{rrrr}
\hline$i$ & $u_{i}$ & $v_{i}$ & $d_{i}$ \\
\hline 1 & 3 & 3 & 0 \\
2 & 11 & 30 & 0 \\
3 & 30 & 109 & 0 \\
4 & 109 & 297 & 0 \\
5 & 297 & 1079 & 0 \\
6 & 1079 & 2940 & 1079 \\
7 & 4019 & 10681 & 297 \\
8 & 10978 & 39784 & 109 \\
9 & 39893 & 108671 & 30 \\
10 & 108701 & 394900 & 11 \\
11 & 394911 & 1076029 & 3 \\
12 & 1076032 & 3909216 & 1 \\
13 & 3909217 & 10651619 & 3909217 \\
14 & 14560836 & 38697260 & 1076031 \\
15 & 39773291 & 144137421 & 394907 \\
16 & 144532328 & 393715003 & 108690 \\
17 & 393823693 & 1430722591 & 39853 \\
18 & 1430762444 & 3898452770 & 10869 \\
19 & 3898463639 & 3623 \\
20 & 14163092112 & 14163088489 & 0 \\
21 & 38590812697 & 38590812697 &
\end{tabular}

5. Asymptotic estimates. As suggested by Gerd Hofmeister, we round off the discussion by giving some asymptotic estimates of the number $g_{\tilde{h}_{0}=h}(k)$, defined in Section 1 (just before Conjecture 1.4). We start by presenting some results of Hofmeister [1], [2] on the extremal regular $h$-range $g_{h}(k)$, from which the results on $g_{\tilde{h}_{0}=h}(k)$ then easily follow.

TheOrem 5.1 (Hofmeister). We have

(a)

$$
\begin{aligned}
& g_{h}(k) \sim\left(\frac{k}{h}\right)^{h} \quad \text { for fixed } h \text { and } k \rightarrow \infty, \\
& g_{h}(k) \sim\left(\frac{h}{k}\right)^{k} \quad \text { for fixed } k \text { and } h \rightarrow \infty, \\
& \text { (c) } \quad g_{k}(k) \sim \frac{\tau}{\sqrt{5}}\left(\tau^{2}\right)^{k} \quad \text { for } k \rightarrow \infty \text {, where } \tau=\frac{1+\sqrt{5}}{2} \text {. }
\end{aligned}
$$

Proof. (a) and (b) follow from formulas (37b) and (37a) in [1, p. 56], respectively.

(c) Using, in the case $h=k$, the $h$-basis coming from (1.10), together 
with results in $[2$, p. 65$]$, we get

$$
g_{k}(k)=\left\lfloor\frac{\tau}{\sqrt{5}}\left(\tau^{2}\right)^{k}\right\rfloor .
$$

Corollary 5.2. We have

$$
\begin{aligned}
& g_{\tilde{h}_{0}=h}(k) \sim\left(\frac{k}{h}\right)^{h} \quad \text { for fixed } h \text { and } k \rightarrow \infty, \\
& g_{\tilde{h}_{0}=h}(k) \sim 2\left(\frac{h}{k-1}\right)^{k-1} \quad \text { for fixed } k \text { and } h \rightarrow \infty,
\end{aligned}
$$

(c)

$$
g_{\tilde{h}_{0}=k}(k) \sim \frac{\tau}{\sqrt{5}}\left(\tau^{2}\right)^{k} \quad \text { for } k \rightarrow \infty .
$$

Proof. (a) If $h<k$, then, by Remark $1.5, g_{\tilde{h}_{0}=h}(k)=g_{h}(k)$.

(b) Suppose that $h>k$. Let $A_{k}=\left\{a_{1}, \ldots, a_{k}\right\}$ be the $h$-basis associated with the coefficients $\mu_{i}^{(1)}$ of (1.10). We show that

$$
g_{\tilde{h}_{0}=h+1}(k+1)=2 g_{h}(k)+a_{k}+1 .
$$

Let $B_{k+1}=\left\{b_{1}, \ldots, b_{k+1}\right\}$ be the basis for which $g_{h+1}\left(B_{k+1}\right)=$ $g_{\tilde{h}_{0}=h+1}(k+1)$. This means that we replace $h$ by $h+1$ and $k$ by $k+1$ in (1.13). Comparing (1.10) and (1.13) we then immediately obtain $\mu_{i}^{(1)}=\mu_{i}$ for $i=1, \ldots, k$ and $\mu_{k+1}=1$, from which it follows that $a_{i}=b_{i}$ for $i=1, \ldots, k$ and

$$
g_{\tilde{h}_{0}=h+1}(k+1)=g_{h}(k)+b_{k+1} .
$$

We have

$$
b_{k+1}=\left(\mu_{k}+2\right) b_{k}-b_{k-1}=\left(\mu_{k}^{(1)}+2\right) a_{k}-a_{k-1}
$$

and therefore, using (2.9), (2.7), (2.11), and (2.12),

$$
g_{h}(k)=\left(\mu_{k}^{(1)}+1\right) a_{k}-a_{k-1}-1=b_{k+1}-a_{k}-1,
$$

which, together with (5.2), implies (5.1).

From (1.12) it follows that $a_{i+1} \leq\left(\mu_{i}^{(1)}+2\right) a_{i}$ for $i=1, \ldots, k-1$, and therefore $a_{k} \leq \prod_{i=1}^{k-1}\left(\mu_{i}^{(1)}+2\right)$. It follows from this (see (2.10), (2.3), and (2.8)) that

$$
a_{k} \leq\left(\frac{h+3 k-1}{k}\right)^{k-1} .
$$

Using (5.1), (5.3), and Theorem 5.1(b), we complete the proof.

(c) We suppose, finally, that $h=k$. Using Remark 1.5 we see that there is nothing to prove, since $g_{\tilde{h}_{0}=k}(k)=g_{k}(k)$. However, we would like to offer a simple alternative proof. Using Remark 1.5 and (1.3) we immediately obtain 
(see formula (35) in $[8$, p. 178])

$$
g_{\tilde{h}_{0}=k}(k)=F_{2 k+1}-1 .
$$

The desired asymptotic estimate then follows from (see formula (62) in $[8$, p. 180])

$$
F_{n}=\left\lfloor\frac{\tau^{n}}{\sqrt{5}}+\frac{1}{2}\right\rfloor \quad \text { for } n=1,2, \ldots
$$

Acknowledgements. I wish to express my sincere gratitude to Ernst S. Selmer for introducing me to Stamp Problems and for his encouragement to solve this particular problem. Moreover, I thank him for carefully reading the manuscript. I thank Gerd Hofmeister for giving me Mrose's thesis [4] in 1985, when I visited Mainz on my way back from Tata Institute, where I made my first efforts to prove Selmer's conjecture. I would also like to thank him for his interest and for his kind help in Section 5.

My special thanks go to Veikko Ennola and Kalevi Suominen, who very kindly inspected various versions of the manuscript. Their comments greatly helped me to improve the presentation, thereby making the paper more accessible to the non-specialist.

\section{References}

[1] G. Hofmeister, Über eine Menge von Abschnittsbasen, J. Reine Angew. Math. 213 (1963), 43-57.

[2] - Über eine Menge von Abschnittsbasen II, Norske Vid. Selsk. Forh. (Trondheim) 39 (1966), 60-65.

[3] —, Asymptotische Abschätzungen für dreielementige Extremalbasen in natürlichen Zahlen, J. Reine Angew. Math. 232 (1968), 77-101.

[4] A. Mrose, Die Bestimmung der extremalen regulären Abschnittsbasen mit Hilfe einer Klasse von Kettenbruchdeterminanten, Dissertation, Freie Universität Berlin, 1969.

[5] O. Perron, Die Lehre von den Kettenbrüchen I, Teubner, Stuttgart, 1977.

[6] H. Salié, Reichweite von Mengen aus drei natürlichen Zahlen, Math. Ann. 165 (1966), 196-203.

[7] E. S. Selmer, The Local Postage Stamp Problem I, Research Monograph, Dept. of Math., Univ. of Bergen, 1986.

[8] S. Vajda, Fibonacci \& Lucas Numbers, and the Golden Section, Horwood, Chichester, and Halsted Press [Wiley], New York, 1989.

DEPARTMENT OF MATHEMATICS

P.O. BOX 4 (HALLITUSKATU 15)

SF-00014 UNIVERSITY OF HELSINKI

FINLAND

E-mail: JUKKA.PIHKO@CSC.FI 